\title{
Super-Enhancement and Control of Amh Expression
}

\author{
David W. Dresser \\ 3IR, Ashworth Laboratories, The University of Edinburgh, Edinburgh, UK \\ Email: david@daviddresser.co.uk
}

Received 7 April 2016; accepted 15 July 2016; published 18 July 2016

Copyright (C) 2016 by author and Scientific Research Publishing Inc.

This work is licensed under the Creative Commons Attribution International License (CC BY). http://creativecommons.org/licenses/by/4.0/

(c) (i) Open Access

\begin{abstract}
In previous work it was shown that mutation of site 1 in the downstream enhancer sequence (DE) led to ablation of enhancement. Mutation of the Wilms tumour factor element $(\mathrm{Wt})$, situated in the Amh promoter between the tata box and the start of translation (TSS), also led to ablation of enhancement. This suggested that these sites may be the anchor points for a specific duplex factor bridging remote DNA elements to the promoter. Mutation analysis of the DNA sequence between sections 1 and 2 of DE was carried out by site directed mutagenesis. It is reported here that site 4 lying between DE1 and DE2, plays a key role in controlling the level of enhancement.
\end{abstract}

\section{Keywords}

Enhancement-, Super-Enhancement, Amh, Gene Expression

\section{Introduction}

AMH (Amh) (Anti-Müllerian hormone), a member of the TGF $\beta$ (BMP) family of growth factors, has been widely studied with regard to its role in sexual development [1]. SMAT-1, a prepubertal line of mouse Sertoli cells [2], transfected with a plasmid where a prokaryotic reporter gene (d2EGFP) is driven and controlled by Amh promoter and enhancer sequences, has helped an understanding of control of gene expression. While the use of such a reporter may be somewhat indirect to the expression of the eukaryotic gene in its normal physiological environment, results obtained using the EGFP reporter largely agree with previous work where Amh expression was measured directly [3]-[7]: thus providing support for the view that reporter gene analysis can make valid contributions to understanding the driving and control of specific gene expression. It is assumed that SMAT cells contain an Amh gene in its normal genomic setting together with a full set of the requisite transcription factors present in a prepubertal Sertoli cell. Figure 1(a) is a summary of key factors thought to influence Amh expression. The role of promoter elements in Amh (EGFP) expression is summarised in the legend to Figure 1(b). 
The prospective downstream enhancer region (DE) was divided into 3 subregions on the basis of a comparison with human AMH.

\section{Methods}

SMAT-1 cells [2] were grown adherently to tissue culture plasticware in DMEM-F12 medium containing glutamax (Gibco), 10\% FCS and antibiotics (penicillin at $10 \mathrm{U} / \mathrm{ml}$; streptomycin at $10 \mu \mathrm{g} / \mathrm{ml}$ ) —for an assay $10^{5}$ cells were established in individual wells of Costar 24-well plates, one day prior to transfection (in the absence of antibiotics) by LipofectAmine 2000 with $800 \mathrm{ng}$ plasmid DNA per well. After a further 2 days of culture the green EGFP expression was measured using a flow cytometer. Fluorescence emission by individual cells was recorded as red and green so autofluorescence could be excluded. Expressed d2EGFP has a half-life of 2 hours in vivo, so measurement is mainly of rate not accumulation. An index of expression was calculated as geometric mean brightness per cell $\left(G_{m}\right) x \%$ cells in the green window.

Site directed mutations were successfully carried out in the promoter region and at DE2 in the enhancer, using the double overlap PCR method [8] [9] with Deep Vent polymerase: this method was consistently unsuccessful when applied to other enhancer sites, using oligo-nucleotide primers based on the sequence shown in Figure 1(b). The "Phusion" method (Thermopol) was successful and has been used for mutation sites 3 - 6 (see Figure 1(b)).

\section{Experiments and Results}

GATA-1 and GATA-4 DNA have opposite effects when added to the transfection mixture which included a plasmid vector with an Amh promoter but lacking any enhancer DNA [10]. Figure 2 shows that a proportionally similar difference is still manifested if the DE enhancer sequence is added to the vector, although the actual overall level of expression is higher. It is clear from the results summarised in this figure that control at the level of the promoter is supplemented and not overridden by enhancement. It seems that the enhancement mechanism is independent of control at the level of transcription factor-promoter interaction: however with other enhancers other interpretations are likely [14] [15].

Previously it was shown that a DNA sequence immediately downstream of the PA signal of a mouse Amh gene, when inserted in an equivalent position in the EGFP plasmid vector, had a moderate enhancer effect on green (reporter) expression [12]. Since it seemed possible that 23 bp of DE between DE1 and DE2, might include a key sequence for enhancement, DNA of this region was made by annealing the appropriate oligo-nucleotides [13] and adding it to the transfection mixture of plasmid constructs with Amh promoter sequences but lacking an intrinsic DE. This double stranded oligo-DNA and the single stranded constituent oligos, were added to the plasmid DNA-Lipofect Amine mixture at the time of transfection of the SMAT cells. The results of these experiments are summarised in Figure 3. High levels of added reagents had a profound (non-specific) inhibitory effect on green expression. A small but significant expression was seen with lower amounts of the reverse oligonucleotide but not with annealed oligo-DNA. Further experiments (Figure 3(b) and Figure 3(c)) confirmed these results and in addition showed that the forward oligo also showed a small but significant incremental effect.

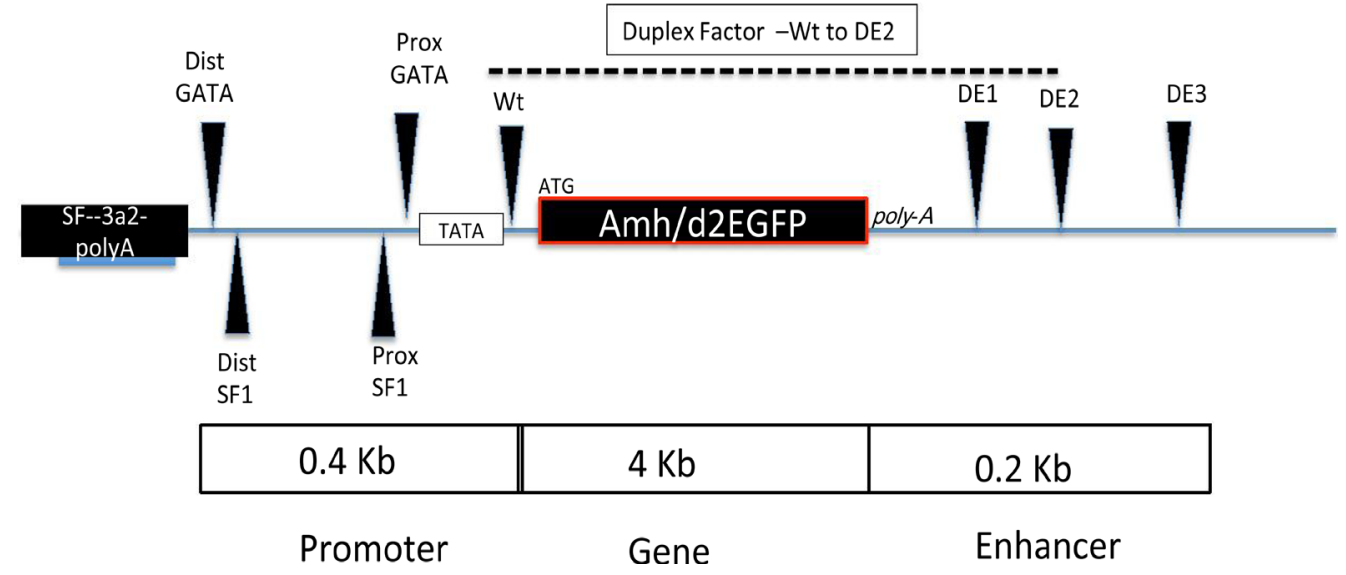

(a) 


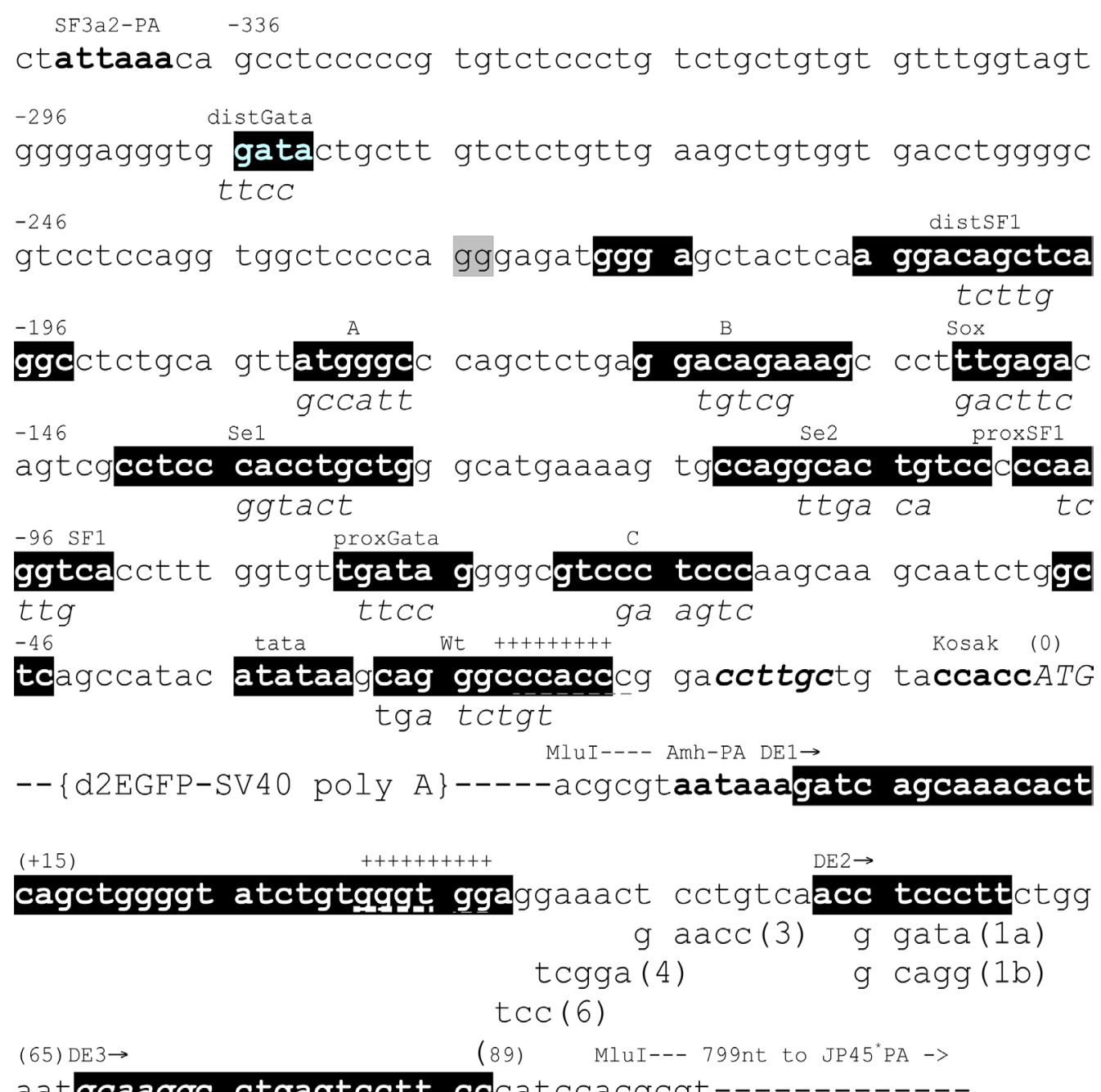

aatgcaaggc ctgagtcctt cccatccacgcgt-------------

* JP45 transcribed in reverse.

(b)

Figure 1. (a) A simplified schematic to illustrate the interelationships between the components of the mouse Anti-Mullerian hormone (Amh) gene. The triangles represent transcription and other elements. The dashed line indicates the Wt-DE2 connection. (b) A detailed sequence map of the Amh gene outlined in (a). Nucleotide sequence (5' to 3') of a mouse Amh promoter and accompanying downstream enhancer sequence. SF3a2-PA is the polyadenylation signal of an upstream gene coding for a spliceosome component. Potential promoter elements (white on black) are identified on the basis of sequence similarity with human and other mammalian Amh promoter sequences: the order of elements is conserved. These potential elements are identified by superscript titles, with mutated sequences indicated as subscripts. Where possible the superscript titles are defined by their affinity for known transcription factors. The start of translation (0) is position 8647 in GenBank mouse genomic nucleotide sequence X83,733. DE is a downstream enhancer starting at the polyadenylation signal for Amh: the DNA for this element was inserted in the d2EGFP vector at a MluI site as indicated in this figure. The MluI site replaces an AfIII site which was in the vector as supplied by Invitrogen. Previously it was shown that mutation of distSF1; sox; Se1; and proxSF1; resulted in a significant reduction in d2EGFP expression [13]. In contrast mutation of proxGata resulted in a small but significant increase in expression [13]. Mutation of the other elements, including the Wilms tumour factor element (Wt) had no measurable effect in this expression system when a potential enhancer was absent. Dual mutation of Wt with any other of the promoter elements had no effect. However when a downstream enhancer (DE) was added to the system, mutation of either Wt or DE2 ablated the enhancer effect [12]: this suggested that these sites are the anchor points for a duplex factor forming a bridge holding the enhancer sequence onto the promoter immediately upstream of the translation start site. A potential short homologous sequence in DE and in Wt is marked by a superscript $+(+++++)$ : the distance between this site and the bridge anchor points is larger in DE than in Wt, suggesting that the DE adherent to the promoter at Wt forms a small bulge or minor loop. 


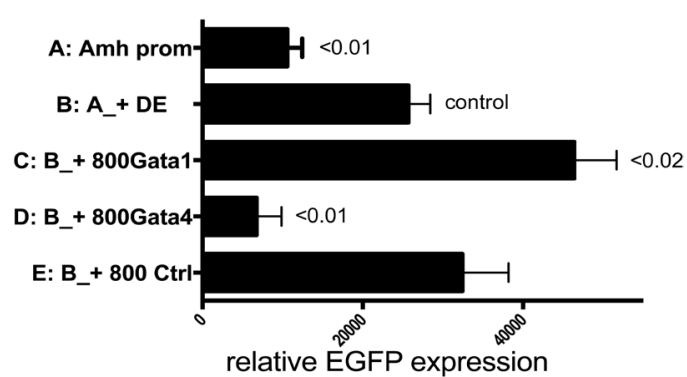

Figure 2. Addition of Gata1 or Gata4 plasmid DNA to the transfection mixture has opposite effects on expression in the absence of a downstream enhancer (DE) [10]. Here it is shown that there is a similar differential effect when there is moderate enhancement due to the presence of intrinsic DE. Ctrl is the DNA of a third party plasmid lacking a Gata insert.... As in all figures p-values are derived from a two tailed t test between control and experimental group, these are included where a positive difference is statistically significant. As in all experiments there are 4 cultures per group. Error bars are SEM. Statistical values calculated using Graph Pad Prism.

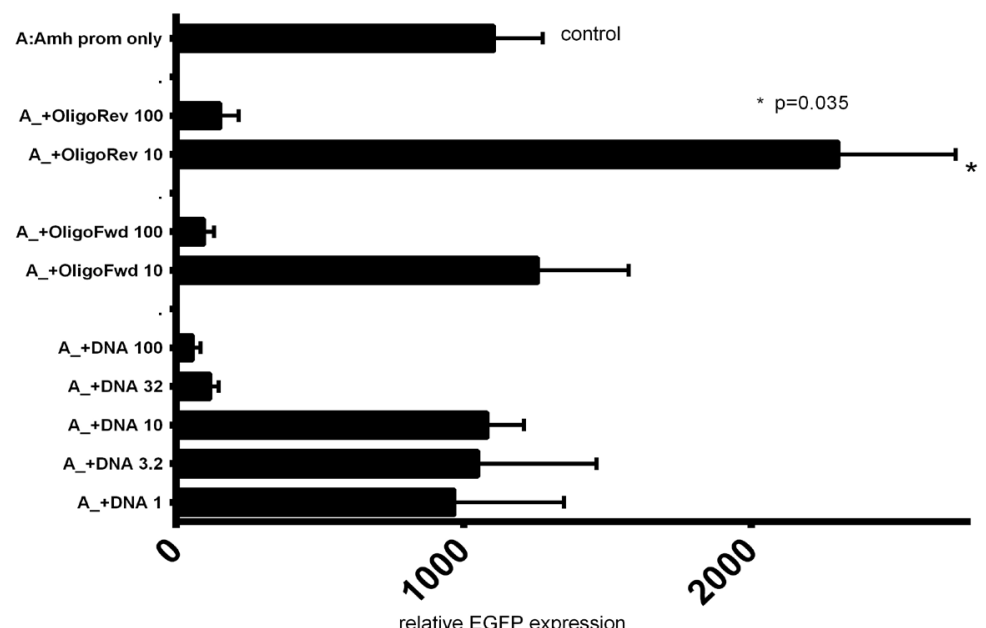

(a)

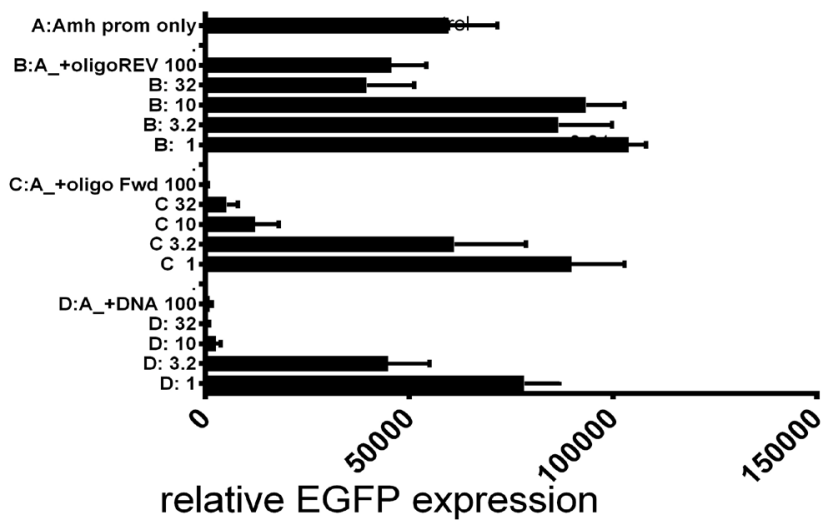

(b)

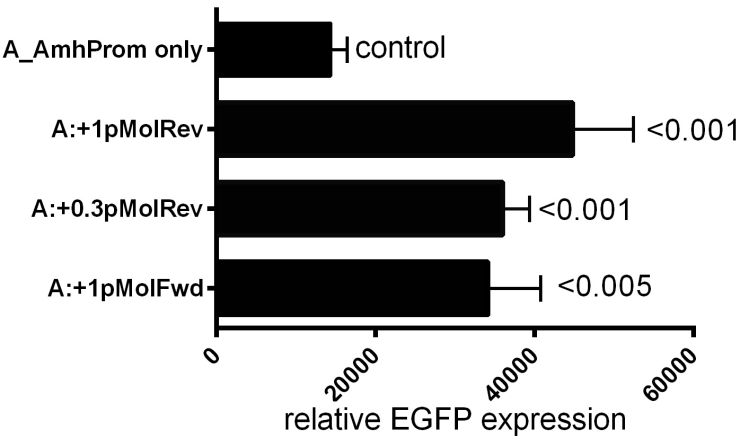

(c)

Figure 3. In the absence of a downstream enhancer (DE): can the putative active enhancer DNA sequence (see Figure 1(b), nt 32 to 55 ) be supplied as free oligo-DNA? The DNA was made by annealing appropriate forward and reverse oligo-nucleotides [13]. The annealed oligo-DNA and its free oligo-nucleotides were added to the transfection mixture 2 days prior to measurement of d2EGFP expression using a flow cytometer. (a): A complete non-specific suppression was observed with quantities of reagent greater than $30 \mathrm{pMol}$ per culture $(0.6 \mathrm{ml})$. With lower amounts there was neither a decrease nor an increase in rate of expression by annealed oligo-DNA. However there was a small but significant incremental effect with 1pMol of the reverse oligo. (b): A repeat of the experiment gave the same result. (c): These results were confirmed in another repeat experiment, with the added result that $1 \mathrm{pMol}$ of forward oligo also gave a significant increase in expression. Comparison of the 10 pMol group in "(a)" and "(b)” suggests that this concentration is at a critical point between non-specific suppression and a specific incremental effect. 
Figure 4(a) and Figure 4(b) illustrate the effect of mutating site 4, (see Figure 1(b), Figure 5) in the prospective minor loop of DE formed when it is closely associated with the Amh promoter. This modification of DE results in "super-enhancement" of EGFP expression. It is also clear that with a simultaneous mutation of Wt superenhancement is ablated, presumably by disruption of the Wt-DE2 bridge. The term "super-enhancer" is used here in the context of the experimental results described-there are other definitions [14].

\section{Discussion}

In plasmid constructs without an enhancer sequence, mutation of the Wilms tumour factor element (Wt), either alone or in combination with mutation of other promoter elements, had no effect. However when DE is present in the construct, mutation of Wt and/or the downstream enhancer element DE2 resulted in ablation of enhancement [12]. These results suggested that the DE sequence was brought into close contact with the promoter immediately upstream of the start of translation, by a specific duplex factor, thus forming a major loop in the genomic DNA, as well as bringing the essential elements for enhancement into close juxtaposition with the promoter.

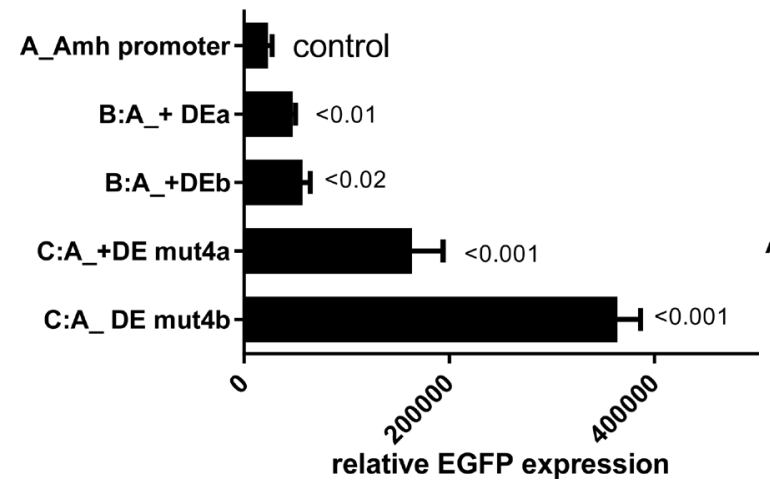

(a)

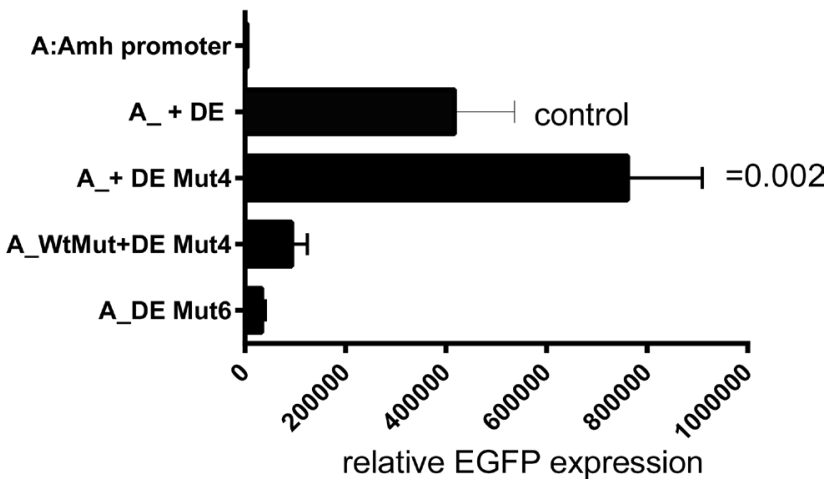

(b)

Figure 4. The consequence of mutating site 4, in the putative minor-loop in DE, when this is bridged to Wt in the promoter, is illustrated in this figure: there is a very large increment in d2EGFP expression in both experimental groups. (a): The increment by unmutated DE is relatively modest but nevertheless significant. The large increment in EGFP expression is due to an increase in the rate of expression by individual cells $\left(\mathrm{G}_{\mathrm{m}}\right)$. Mutations $(1 \mathrm{a}, 1 \mathrm{~b})$ and of Wt in the promoter, ablate the moderate enhancer effect of DE [11]. Mutation of sites 3 and 6 had no effect in this expression system. As mentioned above, mutation of site 4 led to a super-enhancement. (b): A similar result to that illustrated above was obtained in a repeat experiment, where in addition it was shown that breaking the "bridge", by additionally mutating Wt, also ablates super-enhancement.

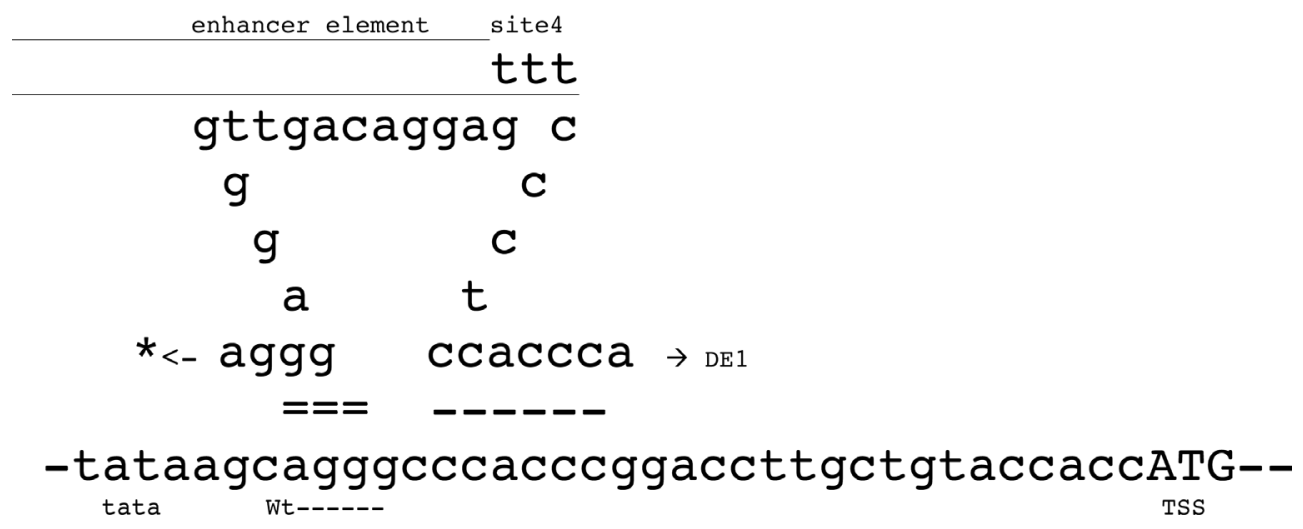

Figure 5. A sequence based diagrammatic representation of the hypothetical juxtaposition of the Amh promoter and the downstream enhancer element (DE). The bottom line is the promoter sequence from the tata box to the translation start site (TSS). *-* represents the gap between the end of the PA signal of the gene and the start of the downstream enhancer: this gap is short in this example but is probably very much greater in other examples of enhancement by remote elements. Superscripted "enhancer element" is an indication of a potential functional sequence which may be responsible for enhancement; "Site4" is the binding site for the brake on (silencer of) super-enhancer activity. = = = represents the anchor sites of a high affinity duplex bridging factor, and - - - - - - is a low affinity sequence homology. 
A reverse identity exists between parts of the $\mathrm{DE}$ and Wt sequences. The gap between the anchor points Wt and DE2, and the homology (сссасc) "sites" are adjacent in Wt but $15 \mathrm{nt}$ apart in DE, suggesting that when DE is bridged (looped) to Wt, there is a bulge or minor loop in the DE DNA. Mutation of site 4 (see Figure 1(b) and Figure 5), which is in this hypothetical minor loop, was made to ascertain if this DNA played any part in enhancement. The results of mutating sites 3 - 6 are summarised in Figure 4.

A sequence consisting of $23 \mathrm{nt}$ immediately upstream of DE2 was put in a NIH Blast search in the mouse genome data base, in particular with reference to chromosome 10; expected hits $<4$; hits achieved $>120$. Since this sequence forms part of the Amh enhancer it seems unlikely to be an example of a random insert of viral origin. Perhaps it is an example of a mechanism forming ordered 3D structure to genomic DNA, which may be related in some way and in some circumstances, to enhancement [15].

Figure 5 is a sequence based schematic illustrating a possible interface of DE2 with the promoter between the tata box and the start of translation. One interpretation of the observation that super-enhancement occurs on mutation of site 4, is that super-enhancement is the default condition, normally down regulated (silenced) by a masking factor or organelle such as a nucleosome-like body [16], specifically binding to this site. Competition for the site by a non-inhibitory factor such as a micro RNA or perhaps an oligo-nucleotide, could result in an easing of control over the release of the brake on the underlying default enhancement mechanism. A similar relaxation of control is achieved by mutating the site. Removal of control of default expression at critical moments in development, such as the removal of the Müllerian duct in early male development [1], would result in a burst of hyper-expression. The high frequency of the occurrence of $\sim 23$ nt upstream of DE2 suggests that this sequence may be associated with a mechanism for imparting an ordered 3D structure to genomic DNA, which may be part of the mechanism for enhancement by distant DNA elements: a phenomenon widely acknowledged in the recent literature [17]. If enhancement is manifested by the addition of signals to the message, triggering its longevity and/or rapid recycling during translation [18], then this might be detectable by mRNA sequence changes during super-enhancement.

\section{Acknowledgements}

I thank Dr. N. di Clemente for providing the SMAT-1 cell line; Prof. Rick Maizels for the benefit of discussion and a critical reading of a draft of this paper; and Yvonne Harcus for help in the laboratory.

\section{References}

[1] Josso, N., Picard, J.Y., Rey, R. and di Clemente, N. (2006) Testicular Anti-Müllerian Hormone: History, Genetics, Regulation and Clinical Applications. Pediatric Endocrinology Reviews, 3, 347-358.

[2] Belville, C., Jamin, S.P., Picard, J.-Y., Josso, N. and Clemente, N. di (2005) Role of Type I Receptors for Anti-Müllerian Hormone in the SMAT-1 Sertoli Cell Line. Oncogene, 24, 4984-4992. http://dx.doi.org/10.1038/sj.onc.1208686

[3] Munsterberg, A. and Lovell-Badge, R. (1991) Expression of the Mouse Anti-Müllerian Hormone Gene Suggests a Role in Both Male and Female Sexual Differentiation. Development, 113, 613-624.

[4] Arango, N.A., Lovell-Badge, R. and Behringer, R.R. (1999) Targeted Mutagenesis of the Endogenous Mouse Mis Gene Promoter: In Vivo Definition of Genetic Pathways of Vertebrate Sexual Development. Cell, 99, 409-419. http://dx.doi.org/10.1016/S0092-8674(00)81527-5

[5] De Santa Barbara, P., Moniot, B., Poulat, F. and Berta, P. (2000) Expression and Subcellular Localization of Sox9, WT1, and AMH Proteins during Early Human Testicular Development. Developmental Dynamics, 217, $293-298$. http://dx.doi.org/10.1002/(SICI)1097-0177(200003)217:3<293::AID-DVDY7>3.0.CO;2-P

[6] Oreal, E., Mazaud, S., Picard, J-Y., Magre, S. and Carré-Eusebè, D. (2002) Different Patterns of Anti-Mullerianhormone Expression, as Related to DMRT1, SF-1, WT1, GATA-4, Wnt-4, and Lhx9 Expression, in the Chick Differentiating Gonads. Developmental Dynamics, 225, 221-232. http://dx.doi.org/10.1002/dvdy.10153

[7] Shepers, G., et al. (2003) Sox8 Is Expressed during Testis Differentiation in Mice and Synerguses with SF1 to Activate the Amh Promoter in Vitro. The Journal of Biological Chemistry, 278, 2816-2818.

[8] Vandevar, M.A., Weiner, M.P., Huttona, C.J. and Batt, C.A. (1988) A Simple and Rapid Method for the Selection of Oligodeoxynucleotide Directed Mutants. Gene, 65, 129-133. http://dx.doi.org/10.1016/0378-1119(88)90425-8

[9] Ho, S.N., et al. (1989) Site-Directed Mutagenesis by Overlap Extension Using the Polymerase Chain Reaction. Gene, 77, 51-59. http://dx.doi.org/10.1016/0378-1119(89)90358-2

[10] Dresser, D.W. (2014) The Transcription Factors GATA-1 and GATA-4 Have Opposite Effects on DNA Expression Driven by an Amh Promoter. American Journal of Molecular Biology, 4, 150-158. 
http://dx.doi.org/10.4236/ajmb.2014.43016

[11] Dresser, D.W. (2013) Interaction between the Wilms Tumour Factor-1 Element in the Promoter of Amh and a Downstream Enhancer Is Required for a Strong Expression of the Gene in Pre-Pubertal Sertoli Cells. American Journal of Molecular Biology, 3, 165-172. http://dx.doi.org/10.4236/ajmb.2013.33022

[12] Dresser, D.W. and Guerrier, D. (2005) Candidate Sertoli Cell Specific Promoter Element for a TGFbeta Family Member (Amh) and a 3' UTR Enhancer/Repressor for the Same Gene. Gene, 363, 159-165. http://dx.doi.org/10.1016/j.gene.2005.08.004

[13] Dresser, D.W. (2012) Mutated Elements of a Complex Promoter (Amh) Can Help to Demonstrate the Role of Certainelements in Controlling Differential Gene Expression. American Journal of Molecular Biology, 2, 351-358. http://dx.doi.org/10.4236/ajmb.2012.24036

[14] Pott, S. and Lieb, J.D. (2015) What Are Super-Enhancers? Nature Genetics, 47, 8-12. http://dx.doi.org/10.1038/ng.3167

[15] van Arensbergen, J., et al. (2014) In Search of the Determinants of Enhancer-Promoter Interaction Specificity. Trends in Cell Biology, 24, 695-702. http://dx.doi.org/10.1016/j.tcb.2014.07.004

[16] Tillo, D., et al. (2010) High Nucleosome Occupancy Is Encoded at Human Regulatory Sequences. PloS One, 5, e9129. http://dx.doi.org/10.1371/journal.pone.0009129

[17] Anderson, R., et al. (2014) An Atlas of Active Enhancers across Human Cell Types and Tissues. Nature, 507, $455-461$. http://dx.doi.org/10.1038/nature12787

[18] Aitken, C.E. and Lorsch, J.R. (2012) A Mechanistic Overview of Translation Initiation in Eukaryotes. Nature Structural \& Molecular Biology, 19, 568-576. http://dx.doi.org/10.1038/nsmb.2303

\section{Submit or recommend next manuscript to SCIRP and we will provide best service for you:}

Accepting pre-submission inquiries through Email, Facebook, LinkedIn, Twitter, etc. A wide selection of journals (inclusive of 9 subjects, more than 200 journals)

Providing 24-hour high-quality service

User-friendly online submission system

Fair and swift peer-review system

Efficient typesetting and proofreading procedure

Display of the result of downloads and visits, as well as the number of cited articles

Maximum dissemination of your research work

Submit your manuscript at: http://papersubmission.scirp.org/ 